\title{
THE SPLITTING CRITERION OF KEMPF AND THE BABYLONIAN TOWER THEOREM
}

\author{
I. COANDĂ AND G. TRAUTMANN
}

\begin{abstract}
We show that the idea used by Kempf (1990) in order to obtain a splitting criterion for vector bundles on projective spaces leads to an elementary proof of the Babylonian tower theorem for this class of bundles, a result due to Barth-Van de Ven (1974) in the rank 2 case and to Sato (1977) and Tyurin (1976) in the case of arbitrary rank. As a byproduct we obtain a slight improvement of the numerical criterion of Flenner (1985) in the particular case under consideration.
\end{abstract}

MSC 2000: 14F05, 14J60, 14D15

The aim of this note is to provide an elementary and short proof of the following Babylonian tower theorem.

Theorem: Let $\mathcal{E}$ be a locally free sheaf on the projective space $\mathbb{P}_{n}$ (defined over an algebraically closed field $k), n \geq 2$. If $m>\Sigma_{i>0} \operatorname{dim} \operatorname{Ext}^{1}(\mathcal{E}, \mathcal{E}(-i))$, then $\mathcal{E}$ cannot be extended to $\mathbb{P}_{n+m}$ unless it is a direct sum of line bundles.

The Babylonian tower theorem for vector bundles on a punctured spectrum of H. Flenner, 2], asserts, in our special case, the same conclusion but for the larger bound

$$
m>\Sigma_{i \in \mathbb{Z}} \operatorname{dim} \operatorname{Ext}^{1}(\mathcal{E}, \mathcal{E}(-i)) .
$$

On the other hand, Kempf, [3], proved that $\mathcal{E}$ cannot be extended to $\mathbb{P}_{n+1}$ if $\operatorname{Ext}^{1}(\mathcal{E}, \mathcal{E}(-i))=0$ for all $i>0$, unless it splits into a direct sum of line bundles. Our proof of the theorem is inspired by Kempf's proof leading us to use infinitesimal neighbourhoods. The idea of Kempf is reflected in the following

Lemma: Let $\mathcal{F}$ be locally free on $\mathbb{P}_{n}, n \geq 2$, let $H \subset \mathbb{P}_{n}$ be a hyperplane of equation $h=0$, and let $H_{i}$ be the $i$-th infinitesimal neighbourhood of equation $h^{i+1}=0$. The linear projection onto $H$ from a point of $\mathbb{P}_{n} \backslash H$ endows $\mathcal{O}_{H_{i}}$ with the structure of an $\mathcal{O}_{H}$-algebra. Then, if for every $i \geq 1$ the exact sequence

$$
o \rightarrow \mathcal{F}_{H_{i-1}}(-1) \stackrel{h}{\rightarrow} \mathcal{F}_{H_{i}} \rightarrow \mathcal{F}_{H} \rightarrow 0
$$

splits as a sequence of $\mathcal{O}_{H}$-modules, $\mathcal{F}$ is a direct sum of line bundles.

\footnotetext{
${ }^{0}$ Research supported by the DFG-Schwerpunktprogramm 1094
} 
Proof. Here and elsewhere $\mathcal{F}_{Y}$ shall denote the restriction of $\mathcal{F}$ to a subscheme $Y$. A splitting of the sequence of $\mathcal{F}_{H_{i}}$ implies that the induced map $\mathrm{H}^{p} \mathcal{F}_{H_{i}}(a) \rightarrow \mathrm{H}^{p} \mathcal{F}_{H}(a)$ is surjective for every $p \geq 0, i \geq 0, a \in \mathbb{Z}$. Using the exact sequence

$$
0 \rightarrow \mathcal{F}(-i-1) \stackrel{h^{i+1}}{\longrightarrow} \mathcal{F} \rightarrow \mathcal{F}_{H_{i}} \rightarrow 0
$$

one deduces that for $p \leq n-2$ and any $a \in \mathbb{Z}$ the map $\mathrm{H}^{p} \mathcal{F}(a) \rightarrow \mathrm{H}^{p} \mathcal{F}_{H_{i}}(a)$ is surjective for large $i>>0$, because $\mathrm{H}^{p+1} \mathcal{F}(a-i-1)=0$ for large $i$. Consequently, also the maps $\mathrm{H}^{p} \mathcal{F}(a) \rightarrow \mathrm{H}^{p} \mathcal{F}_{H}(a)$ are surjective for any $p \leq n-2$ and any $a \in \mathbb{Z}$. It follows that the maps $\mathrm{H}^{p} \mathcal{F}(a-1) \stackrel{h}{\rightarrow} \mathrm{H}^{p} \mathcal{F}(a)$ are injective for $1 \leq p \leq n-1$ and any $a \in \mathbb{Z}$. But $\mathrm{H}^{p} \mathcal{F}(a)=0$ for $a>>0$. Then $\mathrm{H}^{p} \mathcal{F}(a)=0$ for every $1 \leq p \leq n-1$ and $a \in \mathbb{Z}$. By the well known splitting criterion of Horrocks, $\mathcal{F}$ is a direct sum of line bundles.

Before proving the theorem we do some preparations. Let $\mathbb{P}_{n}$ be embedded in $\mathbb{P}=\mathbb{P}_{n+m}$ as the linear subspace $L$ with equations $x_{n+1}=\cdots=x_{n+m}=0$ and let $S=k\left[x_{0}, \ldots, x_{n+m}\right]$ be the homogeneous coordinate ring of $\mathbb{P}_{n+m}$. Let $\mathcal{I}$ denote the ideal sheaf of $L$ and let $L_{i}$ be the $i$-th infinitesimal neighbourhood of $L$ in $\mathbb{P}_{n+m}$ defined by $\mathcal{I}^{i+1}$. Let $\mathbb{P}_{n+m} \backslash L^{\prime} \stackrel{\pi}{\rightarrow} L$ be the central projection, where $L^{\prime}=\left\{x_{0}=\cdots=x_{n}=0\right\}$ is complementary to $L$ with homogeneous coordinate ring $R:=k\left[x_{n+1}, \ldots, x_{n+m}\right]$. Then $\pi$ endows $\mathcal{O}_{L_{i}}$ with the structure of an $\mathcal{O}_{L}$-algebra. Now, as an $\mathcal{O}_{L}$-module,

$$
\mathcal{O}_{L_{i}} \cong \mathcal{O}_{L} \oplus \mathcal{O}_{L}(-1) \otimes_{k} R_{1} \oplus \cdots \oplus \mathcal{O}_{L}(-i) \otimes_{k} R_{i}
$$

where $R_{i}$ are the graded components of $R$, noting that $\pi$ corresponds to the inclusion $k\left[x_{0}, \ldots, x_{n}\right] \hookrightarrow S$. Moreover, the ring structure of $\mathcal{O}_{L_{i}}$ corresponds to the natural multiplication in this decomposition, defined by

$$
\left(f_{p} \otimes r_{p}\right)\left(f_{q} \otimes r_{q}\right)=f_{p} f_{q} \otimes r_{p} r_{q}
$$

This allows to consider the following ideals of $\mathcal{O}_{L_{i}}$. If $J$ is a homogeneous ideal of $R$, then

$$
J^{(i)}:=\mathcal{O}_{L}(-1) \otimes_{k} J_{1} \oplus \ldots \oplus \mathcal{O}_{L}(-i) \otimes_{k} J_{i}
$$

is an ideal subsheaf of $\mathcal{O}_{L_{i}}$. In fact, if $X \subset \mathbb{P}_{n+m}$ is the subscheme defined by the ideal $J S$, then $\mathcal{O}_{L_{i}} / J^{(i)} \cong \mathcal{O}_{L_{i} \cap X}$. In particular, if $J$ is the ideal of a point $p \in L^{\prime}$, then $J S$ defines the linear subspace $P \subset \mathbb{P}_{n+m}$, which is the linear span of $L$ and $p$, and in this case $\mathcal{O}_{L_{i}} / J^{(i)} \cong \mathcal{O}_{L_{i} \cap P}$.

Proof of the theorem. Let $\mathcal{E}$ be as in the theorem and suppose that there exists a locally free sheaf $\mathcal{F}$ on $\mathbb{P}_{n+m}$ such that $\mathcal{F}_{L} \cong \mathcal{E}$. We shall construct a homogeneous ideal $J \subset R$, generated by (at most) $\Sigma_{i>0} \operatorname{dim}_{\operatorname{Ext}^{1}}(\mathcal{E}, \mathcal{E}(-i))$ homogeneous elements, such that for any $i \geq 0$ the short exact sequence

$$
0 \rightarrow \mathcal{F}_{L_{i}} \otimes_{\mathcal{O}_{L_{i}}} \mathcal{I O}_{L_{i}} / J^{(i)} \rightarrow \mathcal{F}_{L_{i}} \otimes_{\mathcal{O}_{L_{i}}} \mathcal{O}_{L_{i}} / J^{(i)} \rightarrow \mathcal{F}_{L} \rightarrow 0
$$


splits as a sequence of $\mathcal{O}_{L^{-}}$modules. Since $m-1 \geq \Sigma_{i>0} \operatorname{dim} \operatorname{Ext}^{1}(\mathcal{E}, \mathcal{E}(-i))$, there exists a point $p \in L^{\prime} \cong \mathbb{P}_{m-1}$ such that the elements of $J$ vanish in $p$. Let $P$ be the span of $p$ and $L$ as in the preparation. We obtain the commutative diagram

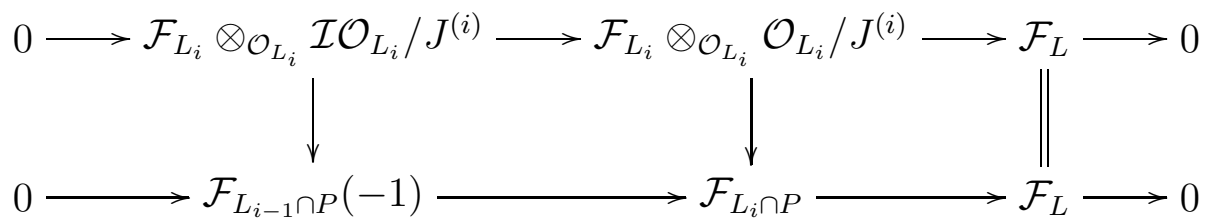

which implies that the bottom row splits, too. Because the schemes $L_{i} \cap P$ are the infinitesimal neighbourhoods of $L$ in $P$, the Lemma implies that $\mathcal{F}_{P}$ is a direct sum of line bundles, and so is $\mathcal{E} \cong \mathcal{F}_{L}$.

As for $J$, it is obtained as the union of an ascending chain of homogeneous ideals of $R$, constructed inductively by a standard technique from infinitesimal deformation theory, adapted to our situation. The induction step is as follows: Let $i \geq 0$ be an integer and assume that an ideal $J \subset R$ has been constructed such that the sequence $\left(A_{j}\right)$ splits for $j \leq i$. Consider the naturally induced diagram

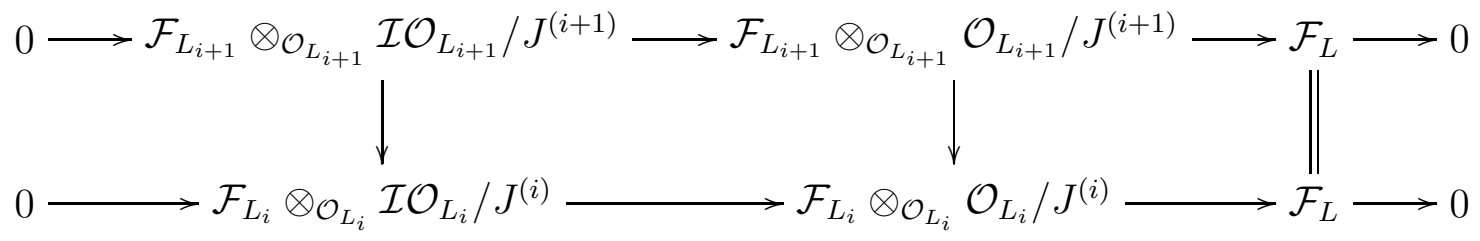

and let $e \in \operatorname{Ext}_{\mathcal{O}_{L}}^{1}\left(\mathcal{F}_{L}, \mathcal{F}_{L_{i+1}} \otimes \mathcal{I} \mathcal{O}_{L_{i+1}} / J^{(i+1)}\right)$ be the extension class (over $\mathcal{O}_{L}$ ) of the top row, which is then mapped in $\operatorname{Ext}_{\mathcal{O}_{L}}^{1}\left(\mathcal{F}_{L}, \mathcal{F}_{L_{i}} \otimes \mathcal{I O}_{L_{i}} / J^{(i)}\right)$ to the extension class of the bottom row, which is supposed to be 0 . Since we have the exact sequence

$$
0 \rightarrow \mathcal{O}_{L}(-i-1) \otimes_{k} R_{i+1} / J_{i+1} \rightarrow \mathcal{I O}_{L_{i+1}} / J^{(i+1)} \rightarrow \mathcal{I O}_{L_{i}} / J^{(i)} \rightarrow 0,
$$

it follows that $e$ is the image of an element $e^{\prime} \in \operatorname{Ext}_{\mathcal{O}_{L}}^{1}\left(\mathcal{F}_{L}, \mathcal{F}_{L}(-i-1)\right) \otimes_{k} R_{i+1} / J_{i+1}$. Let $e_{1}, \ldots, e_{s}$ be a $k$-basis of $\operatorname{Ext}_{\mathcal{O}_{L}}^{1}\left(\mathcal{F}_{L}, \mathcal{F}_{L}(-i-1)\right)$. Then $e^{\prime}=e_{1} \otimes \bar{f}_{1}+\ldots+e_{s} \otimes \bar{f}_{s}$ with $f_{1}, \ldots, f_{s} \in R_{i+1}$. If $K:=J+R f_{1}+\cdots+R f_{s}$, then $K_{j}=J_{j}$ for $j \leq i$, such that the sequences $\left(A_{j}\right)$ for $K$ split for $j \leq i$. In addition,

$$
0 \rightarrow \mathcal{F}_{L_{i+1}} \otimes \mathcal{I O}_{L_{i+1}} / K^{(i+1)} \rightarrow \mathcal{F}_{L_{i+1}} \otimes \mathcal{O}_{L_{i+1}} / K^{(i+1)} \rightarrow \mathcal{F}_{L} \rightarrow 0
$$

splits, too, because of the commutativity of the diagram

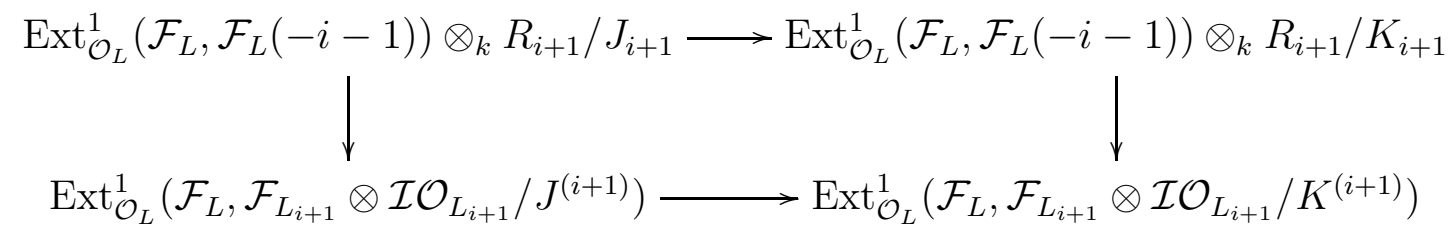

and of the fact that $e^{\prime}$ is mapped to 0 in $\operatorname{Ext}_{\mathcal{O}_{L}}^{1}\left(\mathcal{F}_{L}, \mathcal{F}_{L}(-i-1)\right) \otimes_{k} R_{i+1} / K_{i+1}$. This completes the proof of the theorem. 


\section{REFERENCES}

[1] W. Barth, A. Van de Ven: A decomposability criterion for algebraic 2-bundles on projective spaces, Invent. Math. 25, (1974), 91-106.

[2] H. Flenner: Babylonian tower theorems on the punctured spectrum, Math.Ann. 271 (1985), 153-160.

[3] G.R. Kempf: A criterion for the splitting of a vector bundle, Forum Math. 2 (1990), 477-480.

[4] E. Sato: On the decomposability of infinitely extendable vector bundles on projective spaces and Grassmann varieties, J. Math. Kyoto Univ. 17 (1977), 127-150.

[5] E. Sato: The decomposability of an infinitely extendable vector bundle on the projective space, II, In: International Symposium on Algebraic Geometry, Kyoto 1977, Kinokuniya, Tokyo, 663-672.

[6] A.N. Tyurin: Finite dimensional vector bundles over infinite varieties, Math. USSR Izv. 10 (1976), 1187-1204.

Institute of Mathematics of the Romanian Academy, P.O. Box 1-764

RO-70700 Bucharest, Romania

E-mail address: Iustin.Coanda@imar.ro

Universität Kaiserslautern, Fachbereich Mathematik, Erwin-Schrödinger-Strasse

D-67663 KAISERSLAUTERN

E-mail address: trm@mathematik.uni-kl.de 